\title{
Constructal theory of parabolic scaling
}

\author{
S. Périn \\ OCTO Technology, France
}

\begin{abstract}
The parabolic scaling of rank-size distributions is a very common phenomenon in natural or societal systems such as city sizes, petroleum reservoirs, or galactic intensities. Often, the divergence from a log-log linear power-law is explained by the finite size of the system studied. Several distribution functions were also proposed to best fit this kind of distribution, such as the parabolic fractal or the stretched exponential. In order to explain the emergence of this rank-size distribution pattern, we propose instead to consider a generic mechanism of constructal tree-shaped invasion of a territory combined with the constructal rank-size distribution of a growing flow architecture as the mechanism potentially generating a wide variety of parabolically scaling distributions. Simulations of this mechanism were conducted and we showed that it generated shorter and curved distribution tails similar to a parabolic scaling. In conclusion, we propose to consider the constructal law as the first principle behind the generation of parabolic scaling of rank-size distributions in natural, societal, and engineered systems.

Keywords: constructal law, flow architecture, parabolic scaling, power-law, rank-size distribution, S-curve, Zipf's law.
\end{abstract}

\section{Introduction}

Power-laws and scale-free system architectures are very common in natural or social systems. These phenomena have attracted a strong interested coming from the scientific community, in order to understand and explain the emergence of the scale-free properties of systems.

A frequently encountered property concerns the scale free distribution of the size of the constitutive elements of the system studied, when sorted by considering the rank of these elements relative to their size. This subject is of uttermost interest and some researchers [1] consider that 
"the understanding of the origin of scale invariance has been one of the fundamental tasks of modern statistical physics. How system with many interacting degrees of freedom can spontaneously organize into critical or scale invariant states is a subject that is of up-surging interest to many researcher".

One of the usual and basic tools in this domain of study is the well-known bilogarithmic plot. A log-log plot is particularly interesting to visually check the graph plotted on it, and its tendencies. In the case of a rank-size distribution, the distribution is usually linearly or almost linearly decreasing on the log-log plot on several decades-depending of the size of the sampling.

Often, a power-law distribution is used to fit the distribution. This kind of distribution is a log-log "linear" distribution function of the following form, where $\mathrm{p}>0$ :

$$
\mathrm{f}(x)=k \cdot x^{-p}
$$

Such kind of distribution is identifiable thanks to its linear shape on a log-log graph, where $\mathrm{p}$ dictates the slope of the curve. But, frequently, a divergence on the tail or on the head of the distribution, and sometime on both ends, is noticeable. In the case of the divergence of the tail from log-log linearity, the curvature is frequently assumed as the consequence of the finite size of the system:

"The fact that most of the natural distributions display a log-log curved plot, avoiding the divergence and leading to thinner tails than predicted by a power law, has mostly been interpreted in terms of finite-size effects" [2].

\subsection{Parabolic scaling of rank-size distributions}

This kind of curved distribution on a log-log plot is in fact extremely frequent in different natural or engineered systems such as city sizes, galactic intensities, or petroleum reservoirs [3]. For instance, the rank-size distributions of the source code files of two software frameworks are plotted on fig. 1.

Laherrere proposed to name such distributions Parabolic Fractal (PF), and proposed also to fit them with a polynomial power-law relation as follow [3]:

$$
\ln \left(y_{n}\right)=a \cdot \ln \left(x_{n}\right)^{2}+b \cdot \ln \left(x_{n}\right)+c
$$

with

$$
c=\ln \left(y_{1}\right)
$$

where $y_{1}$ is the ordinate of the first value of the distribution, $a \geq 0$, is the coefficient of curvature, and $b \geq 0$ the slope at the first rank.

Laherrere discusses also the King effect, which is described as the effect explaining, according to him, the frequent divergence of the data with the PF distribution, for the first ranks:

"the new King kills the barons to avoid competition and to acquire a wealth above the commoners" [2].

For instance, the abnormality of the plot of Paris on the log-log distribution of French city sizes is thus explained thanks to this effect [2]. This effect 


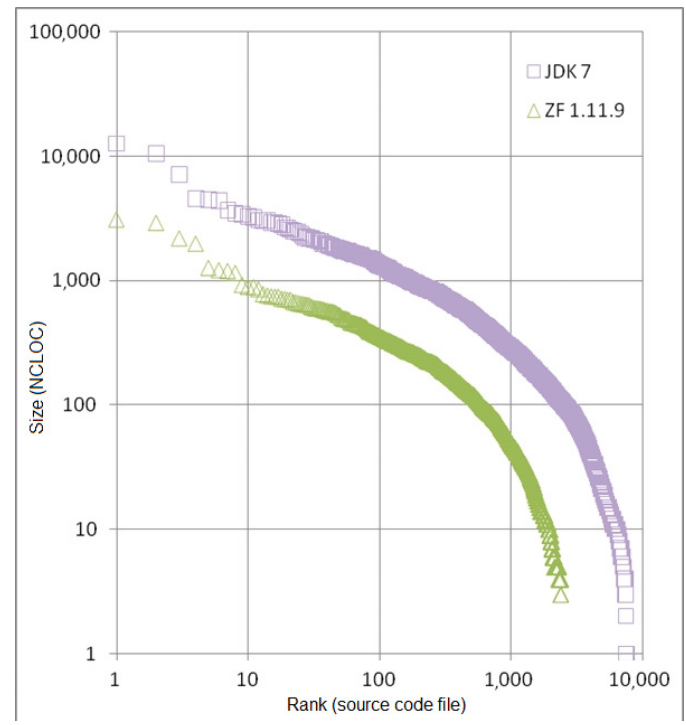

Figure 1: Log-log plot of the rank-size distribution of the NCLOC (Non Comment Lines of Code) metric of the Java and PHP source files, respectively for the Java Development Kit v7 and the Zend Framework v1.11.9.

exacerbates so the concentration of the population in the first ranks. To best fit the data with the model, the first rank must be ignored, in this case, according to the supporters of this idea.

Another alternative model, the stretched exponential, complementary to the power-law and PF models, was also proposed [2]. Usually, power-law, Zipfian distribution, FP, etc., are interpreted as the result of a stochastic growth process, with birth and death mechanism [4]. And, because of the clustering of high-rank values close to the $\mathrm{x}$ axis, values are sometimes grouped into buckets of exponentially increasing size in order to improve the accuracy of the regression [5].

\section{Constructal theory}

For our purpose, we must consider several previous results coming from the field of Constructal Theory, and its first principle, the Constructal Law. Stated in 1996 , the Constructal Law $[6,7]$ is now sustained by a large and increasing body of evidence [8-10]. The Constructal Law states that "for a finite size flow system to persist in time (to live), its configuration must evolve such that it provides easier and easier access to its currents."

The first interesting result for our discussion is the theorization by Bejan et al. of the power-law distribution of city sizes thanks to a constructal architecture of the cities on a landscape [11]. 
Recently, Bejan and Lorente also showed [8] that S-curves can be theorized in terms of a constructal invasion of a territory by a flow system. Tree-shaped invasion were also identified in the same publication as an optimization of the invasion mechanism.

Furthermore, Queiros-Conde et al. [12] showed that a specific constructaltree structure exhibits a parabolic scaling of its geometry.

These results from the Constructal Theory of global optimization under local constraints provided us new keys to better understand the emergence of parabolic scaling rank-size distributions in natural or man-made systems, as detailed hereafter.

\section{Constructal tree-shaped invasion of a territory by a growing population}

In the following paragraphs, we propose to consider a radical new approach to understand the potential mechanism generating such a great variety of distributions ranging from Zipfian and power-law distributions to PF or stretched exponential.

On the basis of the fact that Zipfian, power-law distributions are theoretized by the constructal distribution of city sizes [11], that the logistic (S-curve) function can be predicted thanks to the constructal invasion of a territory [8], and considering that constructal trees can develop a parabolic scaling distribution of their geometry $[12,13]$, we thus propose to consider the generation of curved power-law distribution of the rank-size of a population (such as PF or stretched exponential) as the combination of the constructal tree-shaped invasion of a territory by a growing population or flow, as explained here below.
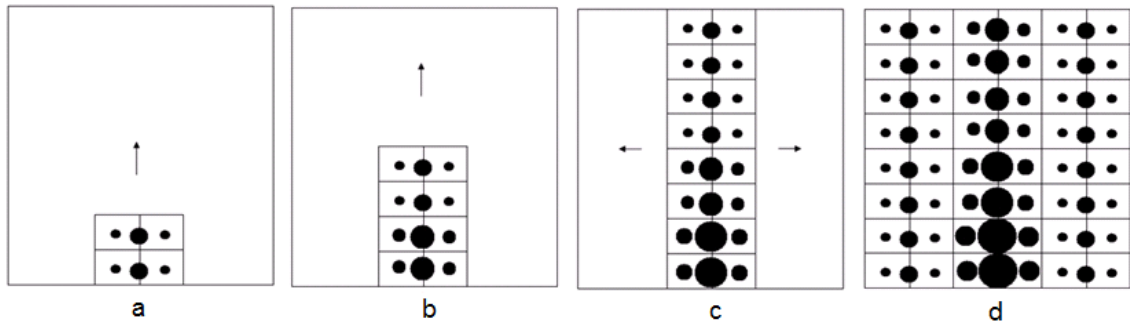

Figure 2: Mechanism of constructal tree-shaped invasion of a territory by a growing flow.

The mechanism is depicted on fig. 2. Initially, a population occupies a small fraction $\left(\mathrm{A}_{0}\right)$ of a bigger territory $(\mathrm{A})$, as showed in fig. 2a. As a hypothesis, the distribution of the population assumes a power-law distribution according to the Constructal Theory documented in [11]. As time progress, the territory colonized by the population extends, and the initial distribution of the population is replicated to another $A_{0}$-sized territory. Now a part $A_{1}=2 . A_{0}$ of the territory is invaded (fig. 2b). Meanwhile, the first population has grown of a factor $\mathrm{g}_{0}$. Then, 
another part of the territory of $\mathrm{A}_{1}$-size is invaded by a new population assuming the same distribution as the initial one (fig. 2c). During the same period, the populations occupying the $A_{1}$ territory have grown of a factor $g_{1}$. This mechanism replicates until the whole A territory is invaded (fig. $2 \mathrm{~d}$ ).

\section{Simulations}

In order to challenge this idea, we run several numerical simulations of this mechanism. These numerical simulations started with hundred initial values, distributed according to a power-law, as showed on fig. 3, and the constructalinvasion mechanism discussed here above was iterated six times, with growth rates of $100 \%$ (doubling). We assumed a power-law on the basis of the results exposed by the constructal theory of distribution of city sizes [11].
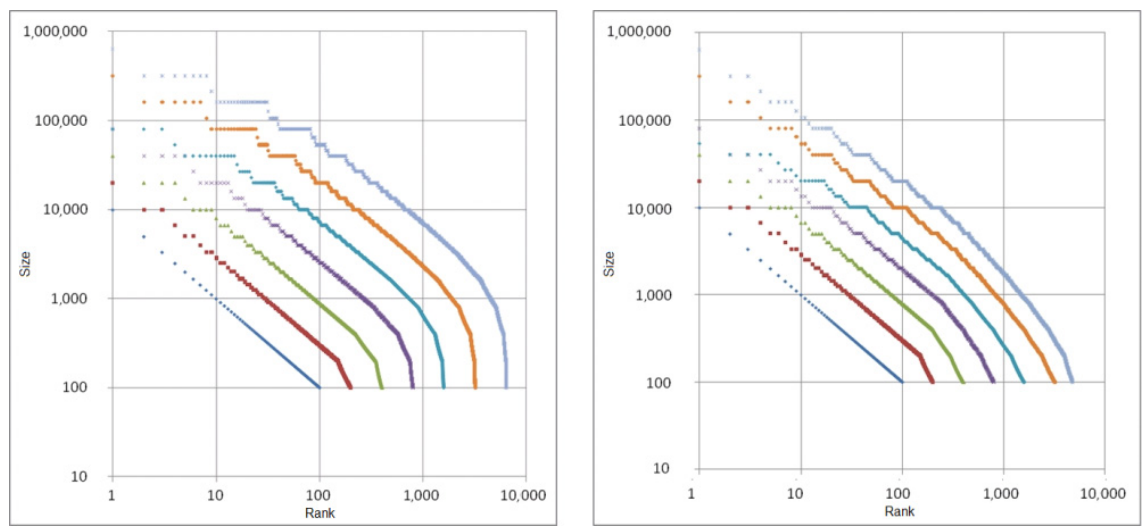

Figure 3: Plots, from the left to the right, of the successive rank-size distributions of a population, for two simulations of the constructal tree-shaped invasion of a territory by a growing flow architecture. In time, the distribution develops a curvature and a shorter tail.

The successive resulting distributions are shown altogether for each of the two graphs displayed on fig. 3. For the simulation whose results are displayed on the left plot, where the curvature is exacerbated, each new generation $\mathrm{N}$ is an replica of the previous generation, whereas, for the plot on the right side, the new $\mathrm{k}^{\text {th }}$ generation $\mathrm{N}$ is composed of $2 \mathrm{k}-1$ replicas of the initial $\mathrm{N}_{0}$ generation.

As expected, the rank-size distributions progressively deviate from the initial, log-log linear, power-law, and develop a curved tail, whereas the first ranks become more stepped.

What is accounted here is the general trend of the distribution resulting from the proposed mechanism, and not the specific fit with a PF, a stretched exponential, or another distribution function. History, local and global factors, heterogeneity, etc., account in natural or man-made system for the uniqueness of 
each rank-size distribution. For instance, it accounts for the "King Effect" in the French cities-sizes distribution [2].

One of the consequences of our theory is the rejection of the assumption that the finite-size effects of a natural system can be accounted for the curvature often encountered in pseudo-power-law distributions [2]. In our proposed mechanism, the main origin of the curvature resides in the conjunction of two dynamics: the growth of older generations, i.e. the upward shift of old plots, and the development of new generations. Because they are more recent, these last start smaller, and tend to accumulate at the end of the distribution (in the long tail), and consequently exacerbating the curvature.

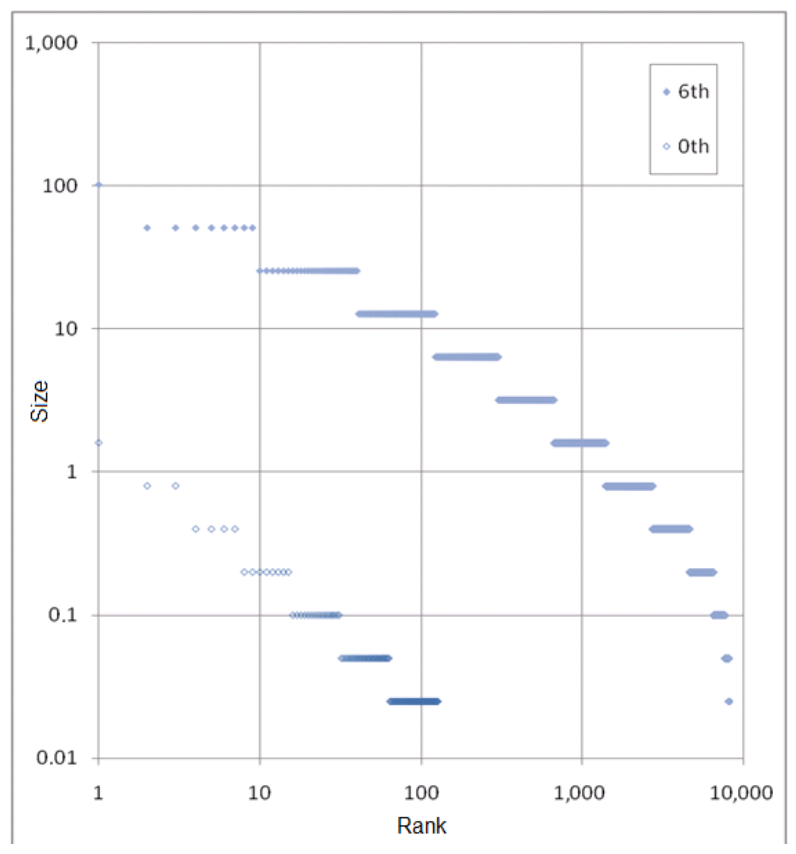

Figure 4: Plot of the initial and sixtieth rank-size distributions of a population, for a 2D simulation of the constructal tree-shaped invasion of a territory.

Similarly, in the here above proposed mechanism, the King Effect results, in a greater upward shift-ie. growth-of the firsts and older concentration points of the distribution, and where the growth is exacerbated. We also ran a $2 \mathrm{D}$ simulation of our mechanism, based on a constructal and planar distribution of a flow architecture over a landscape inspired by Bejan et al. [11]. The resulting distribution and geometry of this simulation are respectively displayed in fig. 4 and 5 .

Despites the fact that we used the term in its spatial acceptation, it must be noticed that the notion of territory used in our model can be extended to any kind of abstract space. 


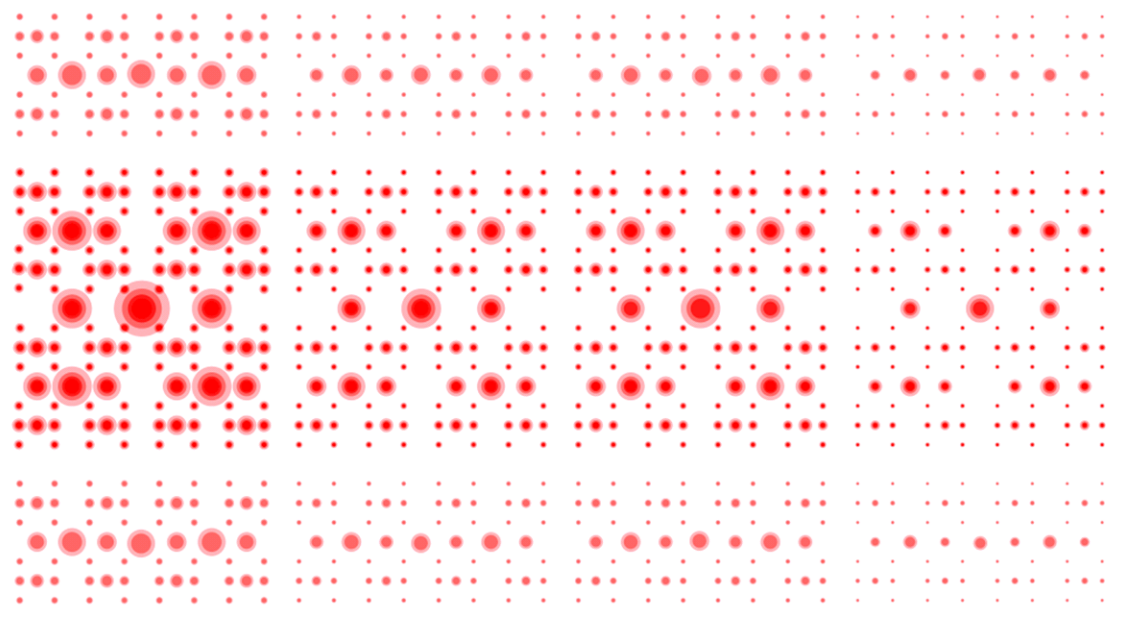

Figure 5: Resulting 2D geometry of a population distribution generated by a simulation of the constructal growth and tree-shaped invasion mechanism (partial view).

\section{Conclusion}

In order to explain the emergence of parabolic scaling of rank-size distributions, we proposed to consider a mechanism of constructal-tree shaped invasion of a territory combined with the constructal distribution of a growing flow as the mechanism potentially generating a wide variety of rank-size distributions, subject to external factors. We showed experimentally, thanks to several numeric and two-dimensional simulations, that this mechanism effectively generates distributions with a shorter, curved tail, similar to a parabolic scaling of a ranksize distribution. It is worth noticing that we don't pretend that our theory accounts for all parabolically scaling distributions, but this mechanism is generic enough to cover a wide variety of phenomena.

In conclusion, we propose to consider the Constructal Law as the main driver behind the generation of parabolic scaling of rank-size distributions in natural or man-made flow systems.

\section{Reference}

[1] Pietronero, L., Tosatti, E., Tosatti, V., and Vespignani, A., Explaining the uneven distribution of numbers in nature: The laws of Benford and Zipf. Physica A 293: 297-304, 2001.

[2] Laherrere J., and Sornette, D., Stretched exponential distributions in nature and economy: "fat tails" with characteristic scales. European Physical Journal B, pp. 2:525-539, 1998. 
[3] Laherrere, J., Distributions de type fractal parabolique dans la Nature. Comptes-Rendus de l'Académie des Sciences - T.322 -Série IIa n7-4 Avril, pp. 535-541, 1996.

[4] Maillart, T. et al., Empirical Tests of Zipf's Law Mechanism in Open Source Linux Distribution. Physical Review Letters, 101(21), p. 218701, 2008.

[5] Wheeldon, R. and Counsell, S., Power Law Distributions in Class Relationships. To appear in Proc. of Third IEEE Int. Workshop on Source Code Analysis and Manipulation, 2003. eprint arXiv:cs/0305037

[6] Bejan, A., Constructal-theory network of conducting paths for cooling a heat generating volume. Int. J. Heat Mass Transfer, 40(4), pp. 799-816, 1996.

[7] Bejan, A., Advanced Engineering Thermodynamics, 2nd ed., Wiley: New York, 1997.

[8] Bejan, A. and Lorente, S., The constructal law origin of the logistics S curve. Journal of Applied Physics, 110(2), p. 024901, 2011.

[9] Bejan, A., Shape and Structure, from Engineering to Nature, Cambridge University Press: Cambridge, UK, 2000.

[10] Bejan, A. and Lorente, S., Design with Constructal Theory, Wiley, 2008.

[11] Bejan, A. et al., Constructal theory of distribution of city sizes (Section 13.4). Advanced Engineering Thermodynamics, 3rd ed., pp. 774-782, 2006.

[12] Queiros-Conde, D. et al., Parabolic scaling of tree-shaped constructal network. Physica A: Statistical Mechanics and its Applications, 384(2), pp. 719-724, 15 October 2007.

[13] Queiros-Conde, D. and Feidt, M., A scale entropy diffusion equation for multi-scale systems: the parabolic case (Chapter X). Constructal theory and multi-scale geometries; theory and applications in energetics, chemical engineering and materials, Les Actes, Les Presses de l'ENSTA: Paris, pp. 117-130, 2010. 